\title{
Web Based Decision Support System for Kidney Exchange
}

\author{
Samy Raja, \\ PG Student, \\ Department of Industrial \\ Engineering \\ Anna University Chennai, \\ India
}

\author{
Prasanna Devi S. \\ Research Scholar, \\ Department of Industrial \\ Engineering \\ Anna University Chennai, \\ India
}

\author{
Suryaprakasa Rao K \\ Professor \\ Department of Industrial \\ Engineering \\ Anna University Chennai, \\ India
}

\begin{abstract}
This paper provides a framework for web implementation of the different matching algorithms for two way kidney paired donation (KPD). The first accept match, weighted Edmond's algorithm and optimized matching algorithm are used to test the matching process in KPD. The results of matching algorithms are obtained in terms of number of matches (transplants) that can be made between incompatible patient donor pairs. Also, currently in India, only cadaver transplant program (CTP) and live related donations are legally acceptable. Hence, this study indicates that the number of patients on waiting list can be enormously decreased, if KPD is legalized in India.
\end{abstract}

\section{INTRODUCTION}

Currently in India, only cadaver program and live related transplantation are legally practiced as per THO Act, 1994 [1]. Because of this, patient has to wait for long time to get a cadaver donor kidney. If the patient remains on dialysis like other treatments, the patient's quality of life drastically reduces. This leads to increase in cost of expenditure as well as death rate. In order to reduce waiting time and death rate, we need to find out the alternative ways of kidney exchange involving live donor mechanism/ methods/ procedures/ algorithm/ combination of more than one. The cadaver kidney program is well established in India. This demands for a good decision support system (DSS) to test run the implementation of live unrelated transplants such as kidney paired donations (KPD) and CTP as well in order to satisfy the demand of kidneys in India. Thus, the objective of this study is to establish a detailed study of various live donor kidney transplantation algorithms implemented across the world and recommend that if KPD is legalized in India, it would drastically reduce the waiting list of patients awaiting transplants.

\subsection{Kidney Transplant Pre-Requisites}

Kidney transplantation is performed only if the patient and the donor organ are compatible by blood type and tissue type. The ABO blood type and tissue type (HLA-Human Lymphocyte Antigen) match are the two characteristics that play key roles in the feasibility and success of a kidney transplant [2]. The first genetic characteristic is the blood type match and the second is the tissue type match. If the matching criteria of Blood group (acceptable blood group, shown in Table 1), and cross match of tissue type (negative cross match) of patientdonor is acceptable, then it is called Compatible pair, else it is called Incompatible pair [3]. The tissue type incompatibility is not considered in most transplants due to the advancement of immunosuppressant drugs.

Table 1 Blood Type Compatibility between Patient and

\begin{tabular}{|c|c|c|c|c|}
\hline Decipient & $\mathrm{O}$ & $\mathrm{A}$ & $\mathrm{B}$ & $\mathrm{AB}$ \\
\hline $\mathrm{O}$ & 1 & 0 & 0 & 0 \\
\hline $\mathrm{A}$ & 1 & 1 & 0 & 0 \\
\hline $\mathrm{B}$ & 1 & 0 & 1 & 0 \\
\hline $\mathrm{AB}$ & 1 & 1 & 1 & 1 \\
\hline
\end{tabular}

In the above Table 1, one indicates compatibility and zero indicates incompatibility patient-donor pairs and Rhtyping of the blood does not play a role in the process of kidney matching.

\section{LITERATURE REVIEW}

Literature review on the implementation of renowned kidney transplantation programs followed across the world are given presented in this section.

1. Cadaver kidney transplantation.

2. Live donor kidney transplantation

- Direct live donor transplantation

- Unrelated or Non-directed live donor transplantation

- Kidney Paired Donation (KPD)

- List exchange 


\subsection{Cadaver Kidney Transplantation Program}

When a patient gets a kidney from a brain dead person within the cold ischemic time, then this type of kidney transplantation is referred in literature as Cadaver kidney transplantation. Under the Cadaver kidney transplantation program, the patient must reach the hospital for achieving transplantation. After the completion of pre-transplant medical check-up, the patient registers for an organ. Whenever there is a cadaver donor organ available, it is matched with list of patients who have registered for a cadaver organ. Eventually the organ is arranged for transplantation to the first patient who achieves a perfect match with the cadaver donor organ. Priority on each patient from the waiting list for the cadaver donor organ is determined by the time spent in the waiting list, as well as blood type match with the donor.

Some potential disadvantages of CTP are:

1. Only a few non-heart beating donors (NHD) or brain stem dead persons are treated as medically acceptable cadaver donors.

2. Number of kidneys donated for transplantation is not sufficient to provide transplants to all those who are waiting.

3. The disparity between the number of donors and potential recipients becomes much wider.

4. Some cadaver kidneys are of marginal qualities which in turn are intentionally rejected by the potential recipients.

5. Graft survival rate is low compared to live donor transplantations.

These disadvantages in Cadaver kidney transplantation are overcome by the live donor kidney transplantation programs.

\subsection{Live Donor Kidney Transplantation Program}

If the kidney to be transplanted is obtained from a person who is alive, then the typical kidney transplantation is referred in literature as live donor kidney transplantation. Donor is a live relative to the recipient. One-year survival rate and one-year graft retention rate is much higher for live donor transplantation than the cadaver transplantation. Living donor kidney grafts have superior survival rates and their availability can also avoid the long waiting time for a cadaver kidney. However, potential living donors can be eliminated from consideration due to incompatibility of the potential donor kidney with the intended recipient. Live donor transplantation represents the most promising solution for closing the gap between kidney supply and demand. Unfortunately, many patients with willing live donors are excluded because of blood type incompatibility or positive donor specific cross match.

\subsubsection{Direct Donor (Relative Donor) or Live Donation}

Under this program, a living donor who is a relative(example spouse, son, father), to the patient, donates his/her kidney to the patient under the waiting list of Cadaver kidney transplantation program that perfectly matches with the donor in blood type and tissue type.

\subsubsection{Unrelated (Non-Directed) Live Donation}

Under this program, a living donor who is not a relative to the patient, donates his/her kidney to the first patient under the waiting list of Cadaver kidney transplantation program that perfectly matches with the donor in blood type and tissue type. The willingness of individual donates organs without a designated recipient is called as non-directed donor.

\subsubsection{Kidney Paired Donation (KPD) Program}

The pair wise kidney donation refers to a recipient patient and his incompatible donor pair. This donor donates his/her kidney to the recipient but issues such that mismatch or incompatible of the blood type, cross matching etc, may prevent the donation from being possible. So this pair is called incompatible pair. If another incompatible patientdonor pair mutually crossover may possible in such that the first patient co-donor can donate a kidney to the second recipient and the second donor can donate to the first recipient [4].

Blood type and cross-match incompatibility will exclude at least one third of patients in need from receiving a live donor kidney transplant. KPD offers incompatible donor/recipient pairs the opportunity to match for compatible transplants. This is the idea behind the kidney pair donation. Under this KPD program there are various methods to exchange the kidneys.

\section{Two way exchange \\ 2. Three way exchange}

We can go up to $\mathrm{n}$ number of ways exchange. Kidney exchange can be done simultaneously between patient and donor. The logistic issue is mainly taken into consideration while possible type of KPD exchange.

\section{Two Way Exchange}

If two patient-donor incompatible pair exchanges their kidney mutually opposite to each other, then the type of exchange is known as two way exchange. Two way exchange are shown in Figure 1, indicates that the first donor1-patient1 incompatible pair because of their HLA mismatch .But blood group is matching patient 1 and its codonor. In the second one Donor2-Patient2 incompatible pair because of blood group mismatch, whereas there is no HLA mismatch. 


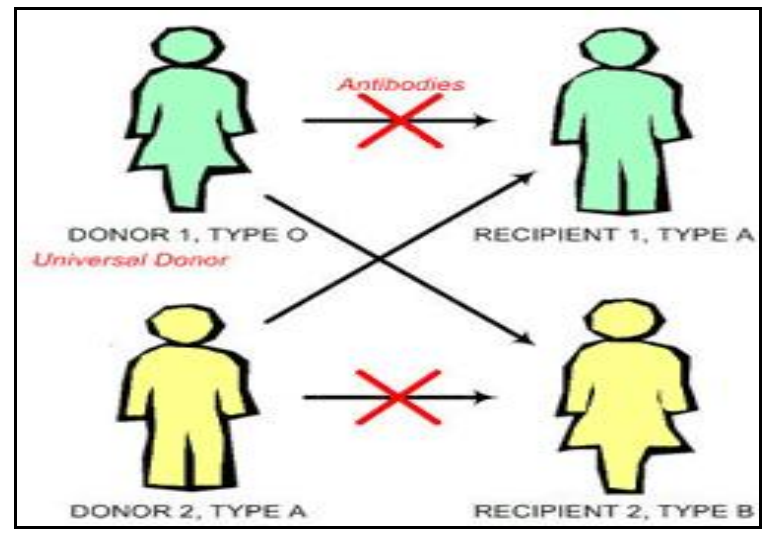

Figure 1 Two-Way Exchange

If these two incompatible patient-donors pair once agreed and willing to exchange their kidneys, based on the compatibility criteria. In figure 1 , the arrow indicates that donor2 match with patient1 and donor1 match with patient2.

If two patient-donor pair is not possible to exchange their kidneys in two ways, those two patients (recipient)-donor pair combined with another patient-donor pair, exchange their kidney is called as three way exchanges.

Likewise, we can go up to "n" way exchange, but the drawback of these " $n$ way" exchange is to ensure logistic and infrastructure facility, since these exchanges are carried out simultaneously. Kidney exchange is likely to proceed incrementally, starting with two way exchange and patient most benefits from incompatible pair. These methods of exchange mainly increase the number of transplantation .The gain from even the small exchanges are large and achievable [5].

\subsubsection{List Exchange Program}

[6] proposed the list exchange (LE) which involves an exchange between one incompatible patient-donor couple, and the cadaver queue. Under this program, an incompatible donor-patient pair approaches the waiting list of patients registered and waiting for an organ under the Cadaver kidney transplantation program [7].

\subsection{Matching Algorithms In KPD}

\subsubsection{First-Accept Match Heuristic}

Step 1: Starts with the first incompatible donor/recipient pair on the list.

Step 2: The database is searched for any donor/recipient pair that meets acceptance criteria as define.

Step 3: If any pair is identified with the acceptance criteria, both patients' donor pair are removed from the database list and transplantation is arranged.

Step 4: These removed pairs are no longer available for consideration in other combinations, even though they might have yield better matches to other pairs.

Step 5: The next first incompatible pair is taken as the starting pair and then it is matched and removed from the list.
Step 6: This process is repeated until all identified matches are performed and no match opportunities remain in the database list.

\subsubsection{Edmond's Matching Algorithm}

The matching of patient - IC donor pair is done mainly based on Edmonds algorithms [8]. In the Edmonds algorithm each node represents an incompatible donorpatient pair. This algorithm considered every feasible solution from the patient -donor pool, compare the solution and pick up the one that best meets a set feasible individualized optimization priorities, modified by predefined optimization bonus.

\subsubsection{Optimization Match}

In matching algorithm all possible combination from entire set are considered at once. Each match in particular feasible solution is given a score based on the optimization priorities of the donor recipient pairs and any optimization bonuses. The score for entire combination of matches are summed. This process repeated for every different feasible solution (different combination of matches possible) for the pool donor recipient pairs. The combination of matches that yields the highest summed score is chosen, these matches are removed from the database and the transplants are arranged.

The evaluation of the kidney transplant programs for different blood group of patients using simulation study has been studied by [9] and reported which program is best suitable for a particular blood type patient. Also, they have reported that irrespective of the blood group type, both KPD and LE programs reduces the waiting time of patients drastically than compared to the CTP. The proposed paper is to implement the development of a web portal DSS to enable the decision maker to optimally match the patient donor pairs, such that the total number of transplants increases. Real time testing of the matching algorithms has been done to illustrate the efficiency of live unrelated transplants.

\section{WEB PORTAL FOR KIDNEY EXCHANGE}

In the simulation model studied by [10], KPD is proved to be better compared to the CTP in terms of reduced waiting time (in days). To further extend, this study intends to demonstrate the effectiveness of implementing KPD as an efficient means to reduce the waiting list of patients (in terms of increasing the number of matches). Hence, this section intends to implement the algorithm for real time testing, by means of developing a web portal, i.e. electronic decision support system (e-DSS) for kidney exchange using C\# and .Net with SQL server as the backend database.

In the real time implementation, the different matching algorithms reported in the literature such as the first accept heuristic, weighted Edmond's and optimized matching have been incorporated into the e-DSS for matching the incompatible pairs.

In the Edmonds algorithm each node represents an incompatible donor-patient pair. This algorithm considers every feasible solution from the patient -donor pool, 
compare the solution and pick up the one that best meets a set feasible individualized optimization priorities.

\subsection{Components of E-DSS}

There are three components in kidney transplantation eDecision support system as shown in Figure 2. They are: Patient-Donor Data Base Management, Model Based Management and User interface Management

\section{(A) Patient -Donor Data Base Management}

Patient -Donor data base management contains two type of data base

\section{Patient - Donor Personal database Management}

2.Patient - Donor medical information system Management

Patient - Donor personal data base management includes Name, Address, Identity number, Agreement for transplantation, Phone number, email id, etc.

\section{DATABASE}

1. Patient-Donor personal information

2. Patient-Donor medical information

\begin{tabular}{|c|c|c|}
\hline $\begin{array}{l}\text { INPUT } \\
\text { 1. Patient- } \\
\text { Donor } \\
\text { (IC) } \\
\text { 2. Patient } \\
\text { Individual } \\
\text { 3. Cadaver } \\
\text { input }\end{array}$ & $\begin{array}{l}\text { 2 WAY KPD } \\
\text { MODEL } \\
\text { BASE } \\
\text { 1. First accept } \\
\text { heuristics } \\
\text { 2. Weighted } \\
\text { Edmonds } \\
\text { 3. Optimized } \\
\text { Match }\end{array}$ & $\begin{array}{l}\text { OUTPUT } \\
\text { Matched } \\
\text { pairs }\end{array}$ \\
\hline
\end{tabular}

Figure 2 Kidney Transplantation e- Decision support System

Patient -Donor medical information system contains, Name, Identity Number, Previous Treatment Information, Blood Group, Antigen Type, Ongoing Treatment Information, Old Test Reports, Ongoing Test Reports, Transplantation Hospital Name, Transplant Hospital Identity Number.

\section{(B) Model Base Management}

The Model Base Management system contain various algorithms such as: Kidney pair donation -two way/ three way, n-way exchanges, First accept match and optimized matching algorithms.

\section{(C) User Interface Management}

User interface management comprises of input and output information. Input having individual patient, individual donor and incompatible and compatible patient donor pairs. Output information supports the Decision maker (Doctor) to make optimal decision on kidney exchange.

The input for the e-DSS implementation follows the following abbreviations. P\# represents Patient identity number. Example P1 represents patient 1 and so on. D\# represents Donor identity number. Example D1 represents patient1's donor (incompatible relative of $\mathrm{P} 1$ ) and so on. BLD represent $\mathrm{ABO}$ Blood Group of patient as well as donor. AGE represent Patients, Donors ages. Example (P1, D1) represents incompatible pair. The tools used for the development of the e-DSS are C\# .Net, for the front end and Microsoft SQL server 2005 is used as the backend database.

\subsection{Development of E-DSS}

Many kidney donor web sites are available today for registering to donate kidney. But these websites are not having patient registration with matching mechanisms. The DSS of web application initially have master data. This master data contains Blood categories, patient, donor and cadaver categories and also have location categories as shown in Figures $3 a, 3 b$ and $3 c$ respectively.

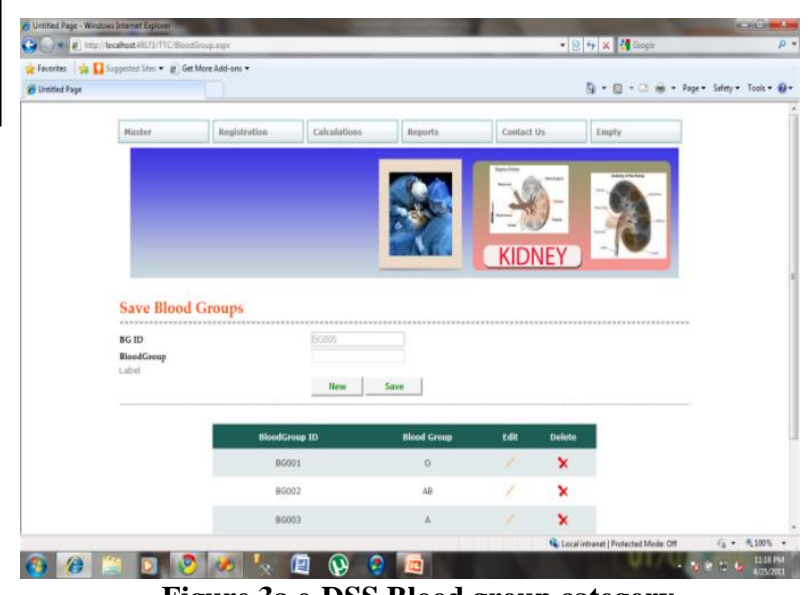

Figure 3a e-DSS Blood group category

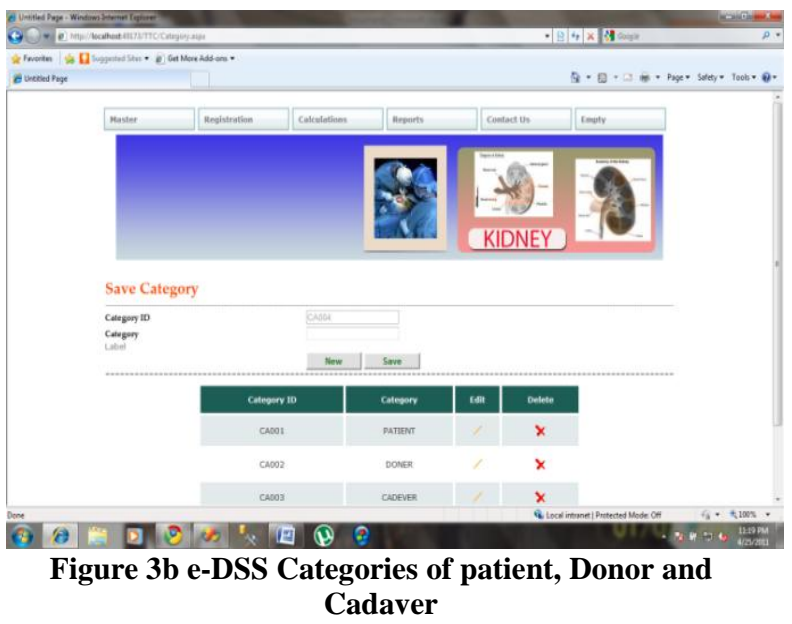




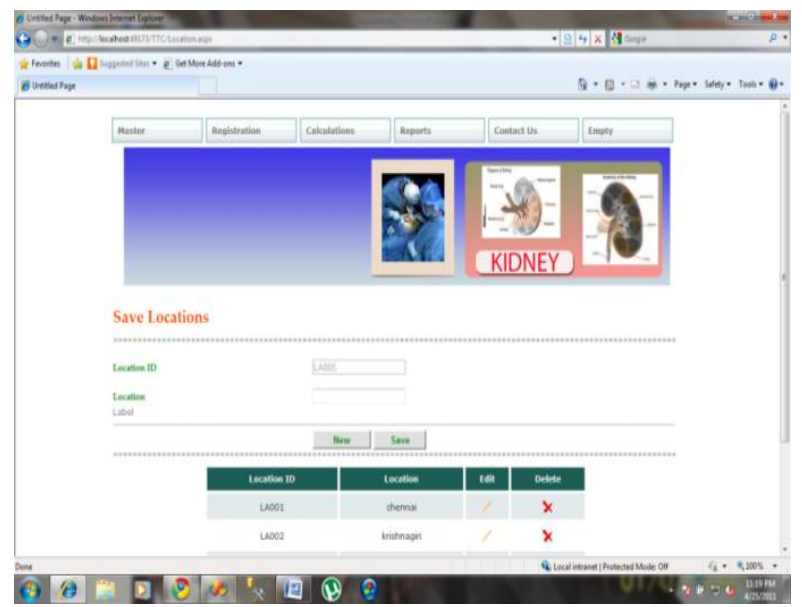

Figure 3c e-DSS locations categories

Registration categories include the patient, donor or the cadaver. The registration form details Include Automatically Generated Identity Number, Category Name, Age, Blood Group, Mail Id, Phone Number, Location, Blood Group, Address etc. List of patient, donor and cadaver report option is also given.

\subsection{Web Application Procedure}

\section{(A) Registration}

The initial step of registration is essential for both the patients and their incompatible donors. The registration forms have Master data like Blood group category, Category type, and location category as specified in previous section. Other than the master data, name, age, mail id, address, date of birth, general comment box and automatically generated registration identity number are the fields of the registration form. The screen shot of the patient registration form is shown in Figure 4.

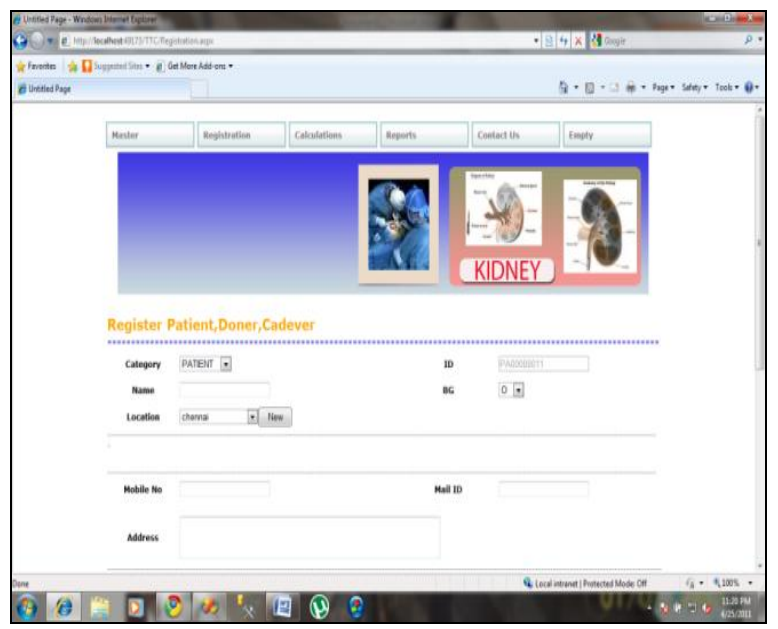

Figure 4 e-DSS Patient registration form

\section{(B) User Login}

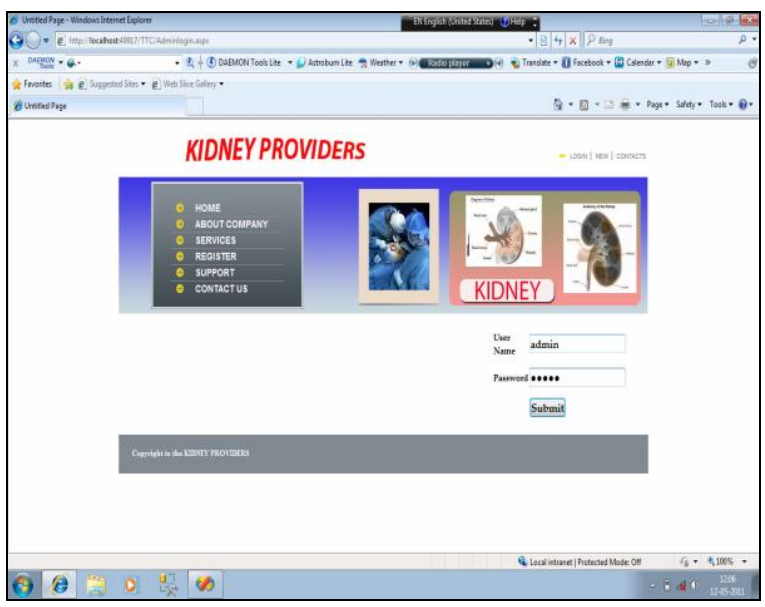

Figure 5 Login Page

User security restricts access to specific user, like patient, donor and cadaver kidney. User security depends on a user identity. After initial registration the user login is used by the patients/ donors/ decision makers to access corresponding information from the web portal as shown in Figure 5.

\section{(C) View Master Data}

The decision makers can access the list of patient details, list of IC donor details at any point of time by accessing the master data - view option, provided in the web portal as shown in Figure 6.

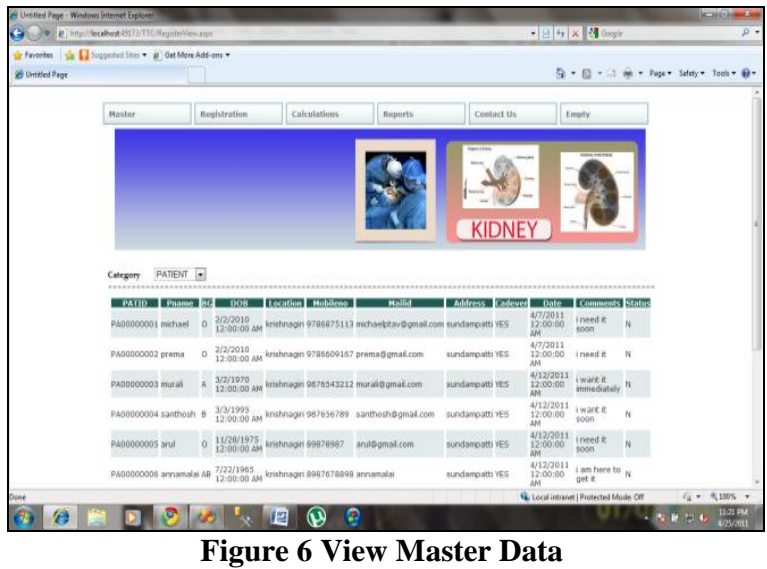

The above Figure 6 shows the Patient list view. The patient list having the details of Patient identity number (PATID), Name of the patient (Name), Date of Birth (DOB), Location, Mobile number, mail id, Address, Registered with CTP ( yes or No), Date of first dialysis and Kidney transplant status etc. If the status is ' $\mathrm{N}$ ' (NO) the kidney is not allocated to the patient and if it is ' $\mathrm{Y}$ ' (Yes) kidney is allocated to the patient. Similarly we can also view for the details of IC Donor list.

\section{(D) Dynamic Allocation in CTP}

In dynamic allocation of patients in CTP, all ESRD patients can register under CTP and the allocation of cadaver organ will be done dynamically based on the blood group, age matching and along with the preference given to the date of first dialysis of the patient. Once the 
allocation of a cadaver organ has been done to the patient, the patient's list and their current status gets automatically updated.

The dynamic kidney allocation is done as and when the cadaver organ arrives. The weight calculation of every patient is done using the formula:

$\mathrm{w} 1 *$ blood type compatibility $+\mathrm{w} 2 *$ age compatibility + w3 $*$ transplant centre preference.

The weights $w 1, w 2$ and $w 3$ are assigned by the transplant coordinator. In this web portal, a weight of $0.6,0.2$ and 0.2 are assigned for $\mathrm{w} 1, \mathrm{w} 2$ and $\mathrm{w} 3$ respectively. The scores for compatibility are shown in Table 2 .

Table 2 Compatibility scores for CTP

\begin{tabular}{|c|c|c|c|}
\hline Criteria & $\begin{array}{c}\text { Highest } \\
\text { Priority }\end{array}$ & $\begin{array}{c}\text { Moderate } \\
\text { Priority }\end{array}$ & $\begin{array}{c}\text { Least } \\
\text { priority }\end{array}$ \\
\hline Blood type & $\begin{array}{c}9 \text { (Exact } \\
\text { match) }\end{array}$ & $\begin{array}{c}6 \\
\text { (Compatible } \\
\text { match) }\end{array}$ & $\begin{array}{c}0 \text { (No } \\
\text { match) }\end{array}$ \\
\hline Age & $\begin{array}{c}9 \\
\text { (difference } \\
\text { of 5 years) }\end{array}$ & $\begin{array}{c}6 \text { (difference } \\
\text { is } \\
\text { Between 6 to } \\
15 \text { years) }\end{array}$ & $\begin{array}{l}3 \\
\text { (difference } \\
\text { more } \\
\text { than } \\
\text { years) }\end{array}$ \\
\hline $\begin{array}{c}\text { Transplant } \\
\text { centre } \\
\text { preference }\end{array}$ & 9 & $\begin{array}{c}6 \\
\text { (Govt) } \\
\text { Aided) }\end{array}$ & $\begin{array}{c}3 \\
\text { (Private) }\end{array}$ \\
\hline
\end{tabular}

The information pertaining to the cadaver organ is fed as input to the web portal and the output obtained is the list of matching patients, in the sorted order of weights/ preferences is obtained as shown in Figure 7.

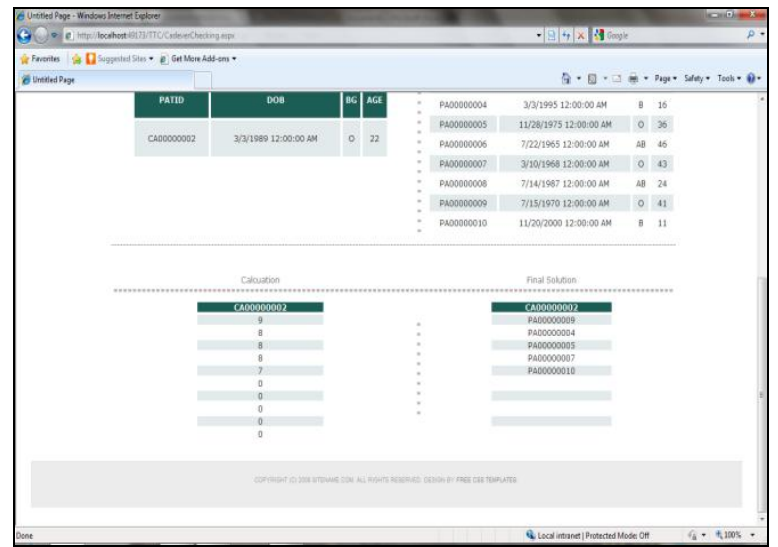

Figure 7 Dynamic Cadaver Allocation Using Web Portal

The final choice of the patient to a particular cadaver organ is made by the decision maker (transplant convener).

\subsection{Implementing Matching Algorithms in KPD}

The list of incompatible pair of patients and corresponding donor can be listed and viewed as shown in
Figure 8. A sample of 10 patient donor incompatible pairs is fed as inputs to the e-DSS and the matches obtained under each algorithm are studied.

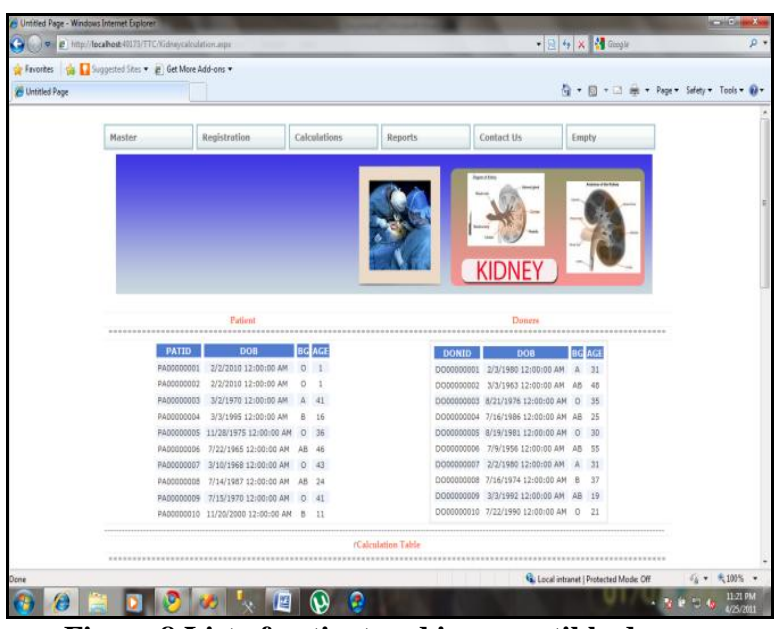

Figure 8 List of patient and incompatible donor

\subsubsection{First Accept Match}

Using first-accept matching heuristic (Segev et al., 2005), the matched pairs are as shown in Figure 9. Only blood type compatibility is considered in this matching algorithm and the preference scores are not taken into consideration. The pairs matched using first accept heuristic algorithm are $(1,5),(2,3),(4,8)$ and $(9,10)$. Thus, a total of $8 / 10(=80 \%)$ pairs are matched using the first accept matching algorithm.

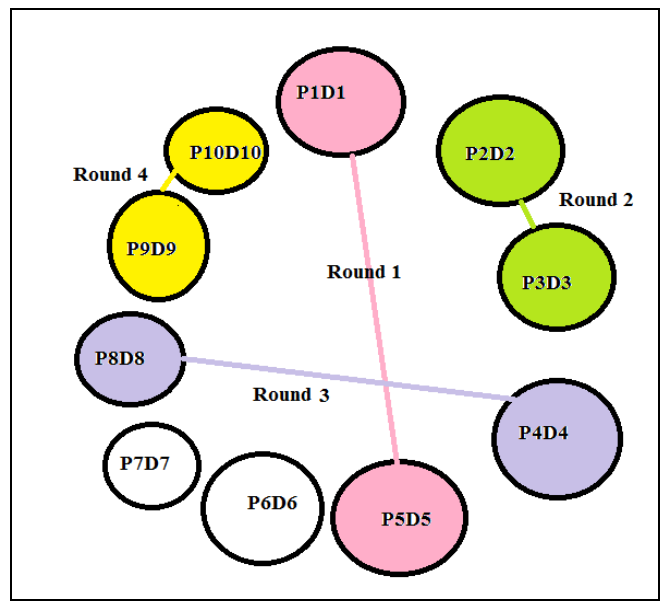

Figure 9 Implementation of First Accept Heuristic in 2way KPD

\subsubsection{Weighted Edmond's Algorithm}

To implement Weighted Edmond's matching algorithm in $\mathrm{KPD}$, all possible matching donors are listed in the order of their compatible preferences for every patient. The preference score is calculated based on the blood group and age compatibility. Figure 10 illustrates the weight based donor listing for every patient (Preference score >3 indicates compatibility, 0 indicates no compatibility). Example patients PA00000004 have three donors. Donor DO00000008 is given first priority because this donor 
weight is 5.5. Similarly Donor DO000000009 is given second priority because this donor weight is 4 and Donor DO0000000010 is given third priority. Like this Donors are prioritized to each patient. From this preference list, the 2way pairing cycles formed in KPD are listed as shown in Figure 11.

The allocation is made in the order of decreasing preference weights. i.e. the pair with highest score gets allocated first, followed by the pairs with decreasing preference score. Ties are broken by randomly choosing among the pairs. Figures 12 and 13 illustrate the implementation of weighted Edmond's algorithm for the test data.

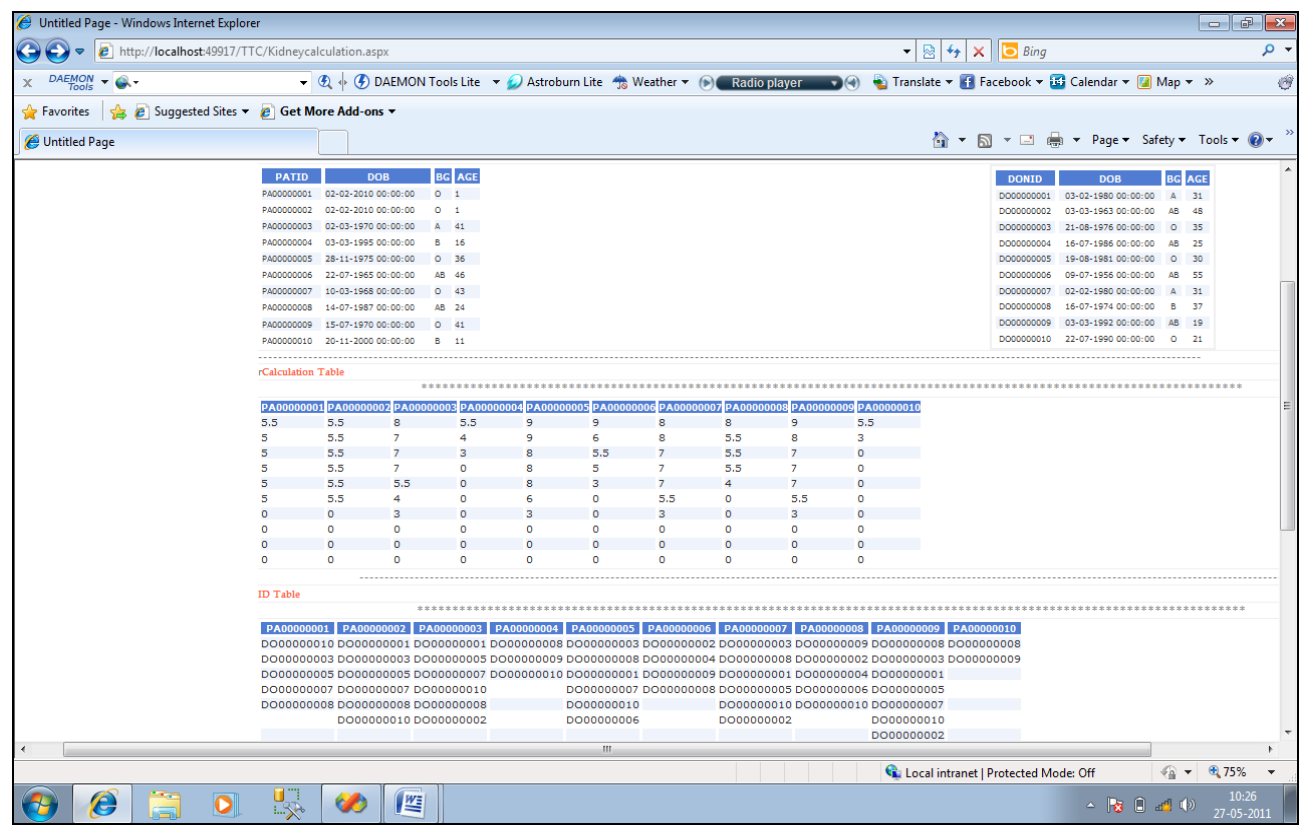

Figure 10 Preferences Listing in 2- Way KPD

\begin{tabular}{cccccccccc} 
pair-1 & pair-2 & pair-3 & pair-4 & pair-5 & pair-6 & pair-7 & pair-8 \\
\hline PA00000003 & PA00000003 & PA00000005 & PA00000005 & PA00000007 & PA00000007 & PA00000007 & PA00000007 \\
\hline DO00000001 & DO00000002 & DO00000003 & DO00000001 & DO00000003 & DO00000001 & DO00000005 & DO000000002 \\
\hline PA00000001 & PA00000002 & PA00000003 & PA00000001 & PA00000003 & PA00000001 & PA00000005 & PA00000002 \\
\hline DO00000003 & DO00000003 & DO00000005 & DO00000005 & DO00000007 & DO00000007 & DO00000007 & DO000000007
\end{tabular}

\begin{tabular}{c|cccccc} 
pair-9 & pair-10 & pair-11 & pair-12 & pair-13 & pair-14 \\
\hline PA00000008 & PA00000008 & PA00000008 & PA00000008 & PA00000009 & PA00000009 \\
DO00000002 & DO00000004 & DO00000006 & DO00000010 & DO00000008 & DO00000010 \\
PA00000002 & PA00000004 & PA00000006 & PA00000010 & PA00000008 & PA00000010 \\
DO0000000 & DO00000008 & DO00000008 & DO00000008 & DO00000009 & DO00000009
\end{tabular}

Figure 11 Two Way KPD Pairing Cycles 


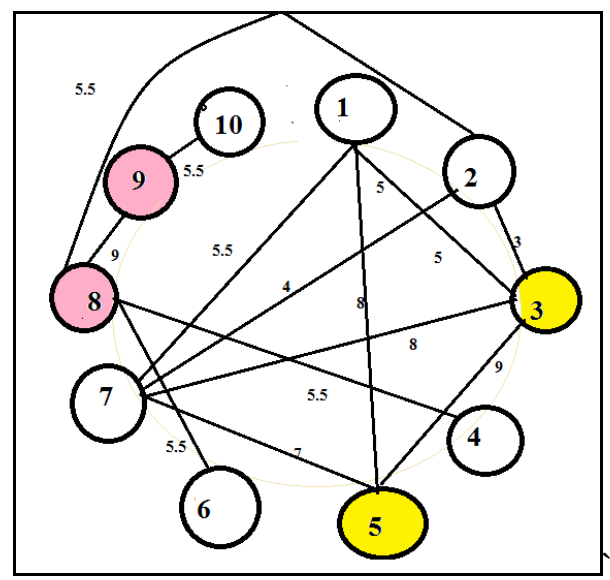

Figure 12 Weighted Edmond's Algorithm: Round 1

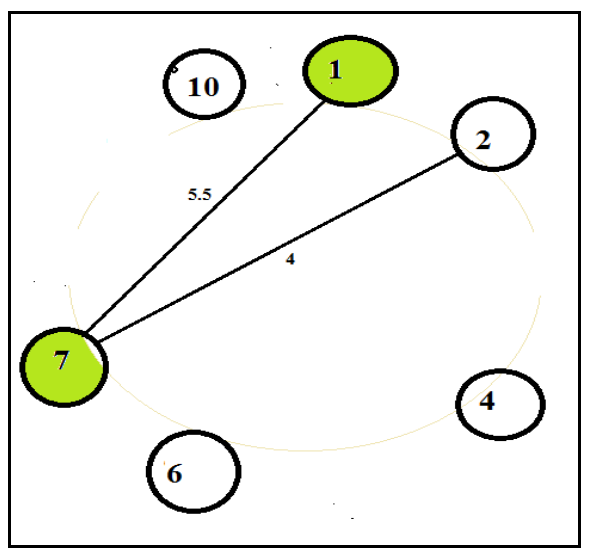

Figure 13 Weighted Edmond's Algorithm: Round 2

In round 1 , pairs $(8,9)$ are matched with highest preference. Hence, the pairs 6 and 4 become disadvantaged due to nonavailability of a matching pair, which is a potential drawback of Edmond's algorithm. The final matching pairs from the implementation of weighted Edmond's algorithm are $(8,9)$, $(3,5)$ and $(1,7)$ respectively, thus producing a matching efficiency of $60 \%$.

\subsubsection{Optimized Matching}

In order to increase the number of matches, the optimized matching algorithm is run to find compatible pair matches. First, the pairs that have the least possible matching links are matched followed by those pairs with increasing matching links. Ties are broken by arbitrarily choosing any one of the links. The results of optimized matching are illustrated in Figures 14 and 15.

In round 1 , the pairs $(10,9),(6,8)$ and $(4,8)$ are candidates with least number of links (equal to one). Since, the compatibility score is same among the 2 pairs $(6,8)$ and $(4$, $8)$, the allocation is done randomly. Thus at the end of round 1 , pairs $(10,9)$ and $(6,8)$ are matched as shown in Figure 7.20 .

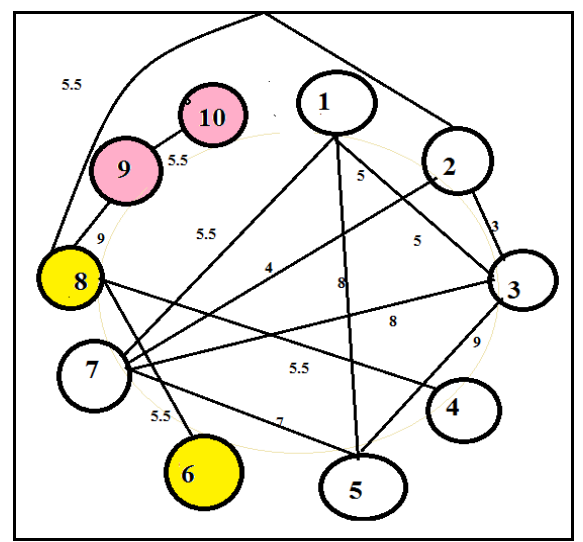

Figure 14 Optimized Matches: Round 1

In the next round, pair 2 has least number of links (equal to two). Hence, the pairs $(2,3)$ and $(2,7)$ are chosen. Among these pairs, $(2,7)$ has higher compatible score compared to $(2,3)$. Therefore pairs $(2,7)$ is allocated as shown in Figure 15.

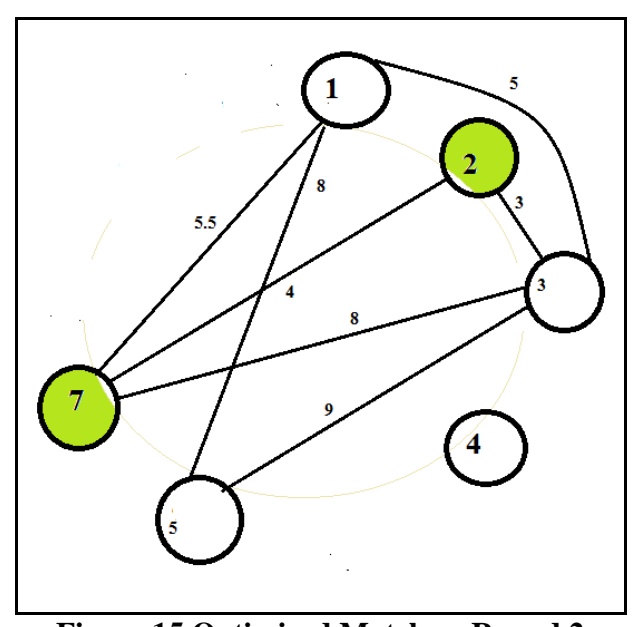

Figure 15 Optimized Matches: Round 2

Among, the left over pairs with least number of links, pairs $(3,5)$ is chosen with maximum compatible score as shown in Figure 16.

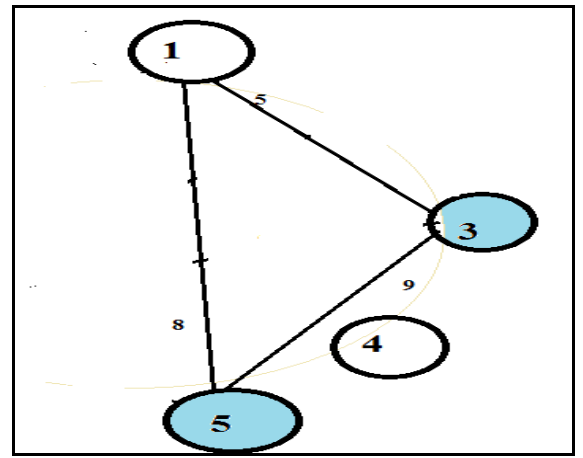

Figure 16 Optimized Matches: Round 3

The number of pairs matched by means of optimized matching are $(10,9),(6,8),(2,7)$ and $(3,5)$. Therefore the matching efficiency of the optimized matching algorithm obtained is $8 / 10$, i.e. $80 \%$. 


\subsection{Real Time Testing}

The data pertaining to the list of kidney failure patients who have come in to government General Hospital, Chennai for a transplant surgery along with their willing donors were considered during a study period of 6 months (May 2010 Nov 2010). Among those patients, around 148 patients have incompatible donors. These patients were sent back, since KPD is not legalized in India. These patients were put to wait under CTP. The number of cadaver (kidneys) arrived during this period is 16 , thus producing a matching efficiency of (16/148) 10\%. Therefore, the input pertaining to the blood group and age of these incompatible patient donor pairs were collected to test the effectiveness of KPD being implemented.
The input data set of 148 patients - IC donor pairs is given as inputs to the e-DSS. The list of matched pairs using all three match algorithms listed above was obtained as outputs. The summary of matching results under different matching algorithms is given in Table 4. It indicates that the percentage increase in the number of matches (compared to CTP) are $59.6 \%, 33.35 \%$ and $48.1 \%$ respectively for the two way exchange program using first accept, Edmond's algorithm and optimized matching algorithms respectively. Hence, it is inferred that if, KPD is legalized in India, the number of matches/ transplants will effectively increase, thus reducing the waiting list of the transplant patients.

Table 4 Summary of Matching Results under e-DSS for Kidney Exchange

\begin{tabular}{|c|c|c|c|c|c|c|}
\hline SI & $\begin{array}{c}\text { KIDNEY } \\
\text { EXCHANGE } \\
\text { PROGRAM }\end{array}$ & $\begin{array}{c}\text { SAMPLE } \\
\text { SIZE }\end{array}$ & $\begin{array}{c}\text { NUMBER } \\
\text { OF } \\
\text { MATCHED } \\
\text { PAIRS }\end{array}$ & $\begin{array}{c}\text { NUMBER OF } \\
\text { UNMATCHED } \\
\text { PAIRS }\end{array}$ & \multicolumn{2}{|c|}{$\begin{array}{l}\text { PERCENTAGE } \\
\text { OF MATCHES }\end{array}$} \\
\hline 1 & $\begin{array}{c}\text { Cadaver } \\
\text { Transplant }\end{array}$ & 148 & $\begin{array}{c}16 \\
\text { (Cadavers) } \\
\end{array}$ & 132 & \multicolumn{2}{|r|}{10.8} \\
\hline 2 & $\begin{array}{l}2 \text { Way KPD - } \\
\text { First Accept }\end{array}$ & 10 & 8 & 2 & 80 & \multirow[t]{2}{*}{$\begin{array}{l}\text { Average } \\
=70.4\end{array}$} \\
\hline 3 & Heuristic & 148 & 90 & 58 & 60.8 & \\
\hline 4 & $\begin{array}{c}2 \text { Way KPD- } \\
\text { Weighted }\end{array}$ & 10 & 6 & 4 & 60 & \multirow[t]{2}{*}{$\begin{array}{l}\text { Average } \\
=44.15\end{array}$} \\
\hline 5 & $\begin{array}{l}\text { Edmond's } \\
\text { Algorithm }\end{array}$ & 148 & 42 & 106 & 28.3 & \\
\hline 6 & $\begin{array}{c}2 \text { Way KPD - } \\
\text { Optimized }\end{array}$ & 10 & 8 & 2 & 80 & \multirow[t]{2}{*}{$\begin{array}{c}\text { Average } \\
=58.9\end{array}$} \\
\hline 7 & Match & 148 & 56 & 92 & 37.8 & \\
\hline
\end{tabular}

\section{CONCLUSIONS}

The web portal for the kidney exchange programs has implemented the first accept, weighted Edmond's and the optimized matching algorithms for two way KPD. The results indicate that the number of matches/ transplants drastically increases if KPD (using any match algorithm) is implemented in India. If the aim of the transplant centre is to maximize the graft survival quality, then Edmond's algorithm can be chosen to be implemented in two way KPD. If a trade off among the graft quality and number of transplants can be allowed, then the optimized match algorithm can be implemented in order to effectively reduce the waiting list of patients.

\section{REFERENCES}

[1] Government of India. Transplantation of Human Organs Act, 1994. Central Act 42 of 1994. Available

from: http://www.medindia.net/tho/thobill1.asp

[2] Rydberg, L. "ABO-incompatibility in solid organ transplantation", Transfus Med., Vol. 11, pp. 325$342,2001$.

[3] Patel, R. and Terasaki, P.I. "Significance of the positive crossmatch test in kidney transplantation". N Engl J Med, Vol.280, pp.735-739, 1969.
[4] Alivn E. Roth, Tayfun Sonmezc, Utku Unverd , A kidney exchange clearinghouse in New England ,The new England journal of medicine , Vol. 95, pp: 376-380, May 2005.

[5] Dorry L.Segev; Sommer E.Gentry; Daniel S.Warren, Kidney paired donation and optimizing the Use of Live Donor Organs., Vol. 293(15), pp: $1883-1890,2005$.

[6] Ross, L.F. and Woodle, E.S. "Ethical issues in increasing living kidney donations by expanding kidney paired exchange programs." Transplantation, Vol.69, pp. 1539-1543, 2000.

[7] Ruth, A.E., Sonmez, T., Unver, M.U, Delmonico, F.L. and Saidman, S.L., "Utilizing List Exchange \& Non-Directed donation through 'chain' paired kidney donations", American Journal of Transplantation, Vol.6, No.11, pp.2694-705, 2006.

[8] Edmonds jack. "Path, Trees, Flowers, "Canadian journal of mathematics, Vol. 17, pp.449-67, 1965.

[9] Prasanna Devi, S., Saravana Kumar, S. and Suryaprakasa Rao, K., "Evaluation of Kidney Transplantation Programs Using System simulation", Journal of medical systems, DOI: 10.1007/s10916-010-9574-0, 2010. 